Full Length Article

Translational Research

\title{
Need for aligning the definition and reporting of cytokine release syndrome (CRS) in immuno-oncology clinical trials
}

\author{
Mark D. Stewart ${ }^{1, *}$, Bruce McCall ${ }^{2}$, Marcelo Pasquini ${ }^{3}$, Allen S. Yang ${ }^{4}$, Carolyn D. Britten ${ }^{5}$, \\ Meredith Chuk ${ }^{6}$, R Angelo De Claro ${ }^{6}$, Bindu George ${ }^{6}$, Nicole Gormley ${ }^{6}$, Mary M. Horowitz ${ }^{3}$, \\ Eric Kowack ${ }^{4}$, Candice McCoy ${ }^{7}$, Phuong Khanh Morrow $^{5}$, Emmanuel Okoye ${ }^{8}$, \\ Rosanna Ricafort ${ }^{7}$, John Rossi ${ }^{9}$, Elad Sharon ${ }^{10}$, Marc Theoret ${ }^{6}$, Ferdinando Vegni ${ }^{7}$, Tai $\mathrm{Yu}^{5}$, \\ Jeff Allen ${ }^{1}$ \\ ${ }^{1}$ Friends of Cancer Research \\ ${ }^{2}$ Genentech, A Member of the Roche Group \\ ${ }^{3}$ Medical College of Wisconsin, Center for International Blood and Marrow Transplant Research \\ ${ }^{4}$ Xencor \\ ${ }^{5}$ Amgen \\ ${ }^{6}$ Food and Drug Administration \\ ${ }^{7}$ Bristol Myers Squibb \\ ${ }^{8}$ Regeneron Pharmaceuticals \\ ${ }^{9}$ CERo Therapeutics \\ ${ }^{10}$ National Cancer Institute
}

\section{A R T I C L E I N F O}

\section{Article History:}

Received 18 October 2021

Accepted 21 January 2022

\section{Keywords:}

Cytokine Release Syndrome (CRS)

Immunotherapies

$\mathrm{T}$ cell receptors

Drug development

\begin{abstract}
A B S T R A C T
As cancer immunotherapies continue to expand across all areas of oncology, it is imperative to establish a standardized approach for defining and capturing clinically important toxicities, such as cytokine release syndrome (CRS). In this paper, we provide considerations for categorizing the variety of adverse events that may accompany CRS and for recognizing that presentations of CRS may differ among various immunotherapies (e.g., monoclonal antibodies, CAR T cell therapies and T cell engagers, which can include bispecific antibodies and other constructs). The goals of this paper are to ensure accurate and consistent identification of CRS in patients receiving immunotherapies in clinical studies to aid in reporting; enable more precise evaluation of the therapeutic risk-benefit profile and cross-study analyses; support evidence-based monitoring and management of important toxicities related to cancer immunotherapies; and improve patient care and outcomes. These efforts will become more important as the number and variety of molecular targets for immunotherapies broaden and as therapies with novel mechanisms continue to be developed.

(c) 2022 Published by Elsevier Inc. on behalf of International Society for Cell \& Gene Therapy. This is an open access article under the CC BY-NC-ND license (http://creativecommons.org/licenses/by-nc-nd/4.0/)
\end{abstract}

\section{Introduction}

The emergence of cancer immunotherapies has led to transformational advances across solid and hematologic malignancies, bringing new hope to patients with serious, life-threatening diseases. Cancer immunotherapies provide clinically beneficial alternatives and additions to traditional cytotoxic treatments. Recent U.S. Food and Drug Administration (FDA) approvals of immunotherapies and the rapid

\footnotetext{
* Corresponding author:

E-mail address: mstewart@focr.org (M.D. Stewart).
}

expansion into new indications for existing agents are enabling broader availability of immunotherapies to cancer patients.

The immuno-oncology (IO) drug development pipeline continues to grow, and cancer immunotherapies are quickly being integrated into the standard of care for many cancers [1]. Importantly, our increasing clinical experience with these immunotherapeutic agents has brought greater awareness to several toxicities unique to immunotherapies that are not typically observed with traditional cytotoxic agents. With the success of newer immunotherapies such as $T$ cell engagers and chimeric antigen receptor (CAR) $\mathrm{T}$ cells in several hematologic malignancies, there has been growing recognition of cytokine release 
syndrome (CRS) as a distinct clinical entity. Cytokine release syndrome represents one of the most common toxicities of these therapies and occurs with varying frequency, severity and presentation among immunotherapeutic agents [2]. The incidence of CRS is relatively low for conventional monoclonal antibodies, but there is a higher risk of CRS with CAR T cell therapies and T cell engagers (incidence ranging from $17 \%$ to $94 \%$ for all grades) [3]. Early in the development of immunotherapies, the term CRS was used more generally to describe a syndrome with a dramatic presentation requiring intensive care, but we now understand that CRS presents with a spectrum of severities, ranging from a selflimited low-grade fever to serious multiorgan collapse.

Although CRS is increasingly recognized as an on-target effect associated with CAR T cells and T cell engagers, the full extent of this syndrome, including pathophysiology and effects on end-organ function, has not been fully characterized. A standardized approach is needed for diagnosing CRS and its manifestations in clinical trials and for reporting CRS in both prescribing information and published literature. In addition, with the advent of $\mathrm{T}$ cell engagers and other IO agents, there is an increasing need to distinguish CRS from other clinical entities, such as acute infusion-related reactions (IRR), septic shock, or hemophagocytic lymphohistiocytosis (HLH). For instance, acute IRRs and CRS can have overlapping symptoms and temporality, but likely have different pathophysiology and differ in management and prognosis. Our current mechanistic understanding of these overlapping clinical entities continues to evolve, and concerted efforts to harmonize data capture will help better characterize these events to uncover key differential features and inform development of individualized mitigation strategies, as appropriate. The importance of capturing adverse events (AEs) in a systematic and harmonized manner has been highlighted by the emergence and growing recognition of immune-effector cell-related AEs observed with immune checkpoint inhibitors and their management, which has been a focus in recent clinical guidelines published by the Society for Immunotherapy in Cancer and the American Society for Clinical Oncology [4,5]. Inconsistent or inadequate characterization of these toxicities in clinical trials impact how data are presented in publications and prescribing information, potentially resulting in suboptimal representation of these clinical events. This, in turn, can put patients at risk if their treatment side effects are not appropriately managed.

\section{Growing Clinical Experience of Infusion Reactions and CRS}

Adverse events known broadly as IRRs have long been defined, diagnosed and reported in an ambiguous and inconsistent manner [6]. This arises, in part, from the fact that the term IRR came into use at a time when few biological therapies were available and acute reactions to an infusion of a biologic agent were starting to be reported. Additionally, little was known about the exact mediators involved in these reactions. Since the introduction of therapeutic monoclonal antibodies and other biologics into clinical practice, IRR has been used as a broad term to encompass acute findings during or shortly after an infusion that may include hypersensitivity/anaphylaxis, complement activation-related pseudoallergy (CARPA), CRS, or more nonspecific signs and symptoms [6]. During clinical development, IRRs are generally defined as AEs occurring within the first 24 $\mathrm{h}$ after infusion of a therapy, with causality deemed by the investigator to be related to the therapy. This operational definition has resulted in the term IRR being used to define a wide array of symptoms with potentially disparate pathophysiology whose main commonality is occurrence within $24 \mathrm{~h}$ of infusion. The majority of IRRs reported with therapeutic monoclonal antibodies are self-limited and treated symptomatically [7-10]. Infusion-related reactions after CAR T cell administration are infrequent and generally mild. Nevertheless, with the emergence of T cell-engaging therapeutics, in particular T cell engagers and other IO agents, distinguishing CRS from
IRR has been a challenge, in that the signs and symptoms may partially overlap.

CRS is a supraphysiologic response driven by the immune system. It has been commonly observed in sepsis and other infections, including those related to COVID-19, and as an on-target AE of T cell-mediated therapies or in response to other therapies such as COVID-19 mRNA vaccines [11]. CRS is initiated by the activation of T cells and mediated by cytokines produced by macrophages and other myeloid cells. CRS can occur within several hours to days after infusion of an immunotherapeutic, but typically does not present beyond 14 days after initiation of therapy [12]. CRS can be short-lived, but often lasts for several days. Because symptoms of CRS can overlap with other toxicities that have generally been classified as IRRs, and because both CRS and IRR can occur within a day after infusion, careful examination of the signs and symptoms, their attribution, and the response to therapy is important. The presentation of CRS may differ depending on the immunotherapeutic and the clinical and biological status of the patient. Factors (therapy- and patient-dependent) include tumor antigen target, location of tumor (i.e., blood vs. solid tumor) and target antigen or T cell binding potency. In addition, the timing of the onset of CRS can coincide closely with infusion of T cell engagers. However, for cellular products, $\mathrm{T}$ cell expansion precedes the onset of CRS, and there is therefore a lag between infusion and CRS symptom onset [13].

CRS typically presents with a fever and may progress to hypotension or hypoxia. Flushing and rash may accompany both anaphylactic reactions and CRS, although specific skin and mucosal changes such as hives and mucosal swelling predominate in anaphylactic reactions, occurring in $80 \%$ of cases [14]. An underlying hallmark associated with CRS is the release of cytokines, and this has been identified as a differentiating criterion in the Common Terminology Criteria for Adverse Events (CTCAE) v.5 definition for CRS and IRRs. However, the measurement of cytokines is not yet a routine element in clinical practice, nor are there reliable cytokine thresholds for CRS diagnosis. Thus, this distinction alone may not yet be helpful to clinicians at the bedside, and emergent interventions are still largely based on the clinical manifestations and severity of CRS as well as response to therapeutic interventions. For example, the role of the interleukin (IL)-6 pathway in CAR T cell therapy has been characterized, and therefore use of IL-6 blocking agents has become a mainstay interventional treatment of CRS $[15,16]$.

\section{CRS Definition and Severity}

In light of our evolving clinical experience with emerging immunotherapeutics, several efforts have been made to update and harmonize grading criteria for CRS in clinical trials (Table 1). Additionally, the elements described in each grading system offer information on what defines severity.

Fever is a CRS-defining characteristic but does not dictate the severity of CRS. Therefore, the Memorial Sloan Kettering Cancer Center (MSKCC) grading system initially relied on the availability of cytokine levels measured from patients in real time to distinguish severe versus nonsevere CRS [23]. However, real-time cytokine testing may be limited to specific health care research settings, and there is currently poor correlation between pre-/posttreatment cytokine levels and the severity of CRS signs and symptoms. Thus, the presence and severity of hypotension and hypoxia are most commonly used to assign the grade of severity for CRS, as these two events typically drive the need for higher level of care (e.g., intensive care) and clinically relevant sequelae. One unique aspect of CRS grading is that the severity is often based on the type and/or level of practitioner intervention. For example, the utilization of one versus more than one vasopressor agent to treat hypotension, or the use of supplemental oxygen alone versus mechanical ventilation for hypoxia, determines the CRS severity grade in several of the currently used CRS grading 
Table 1

Evolving definitions and criteria for grading and managing CRS.

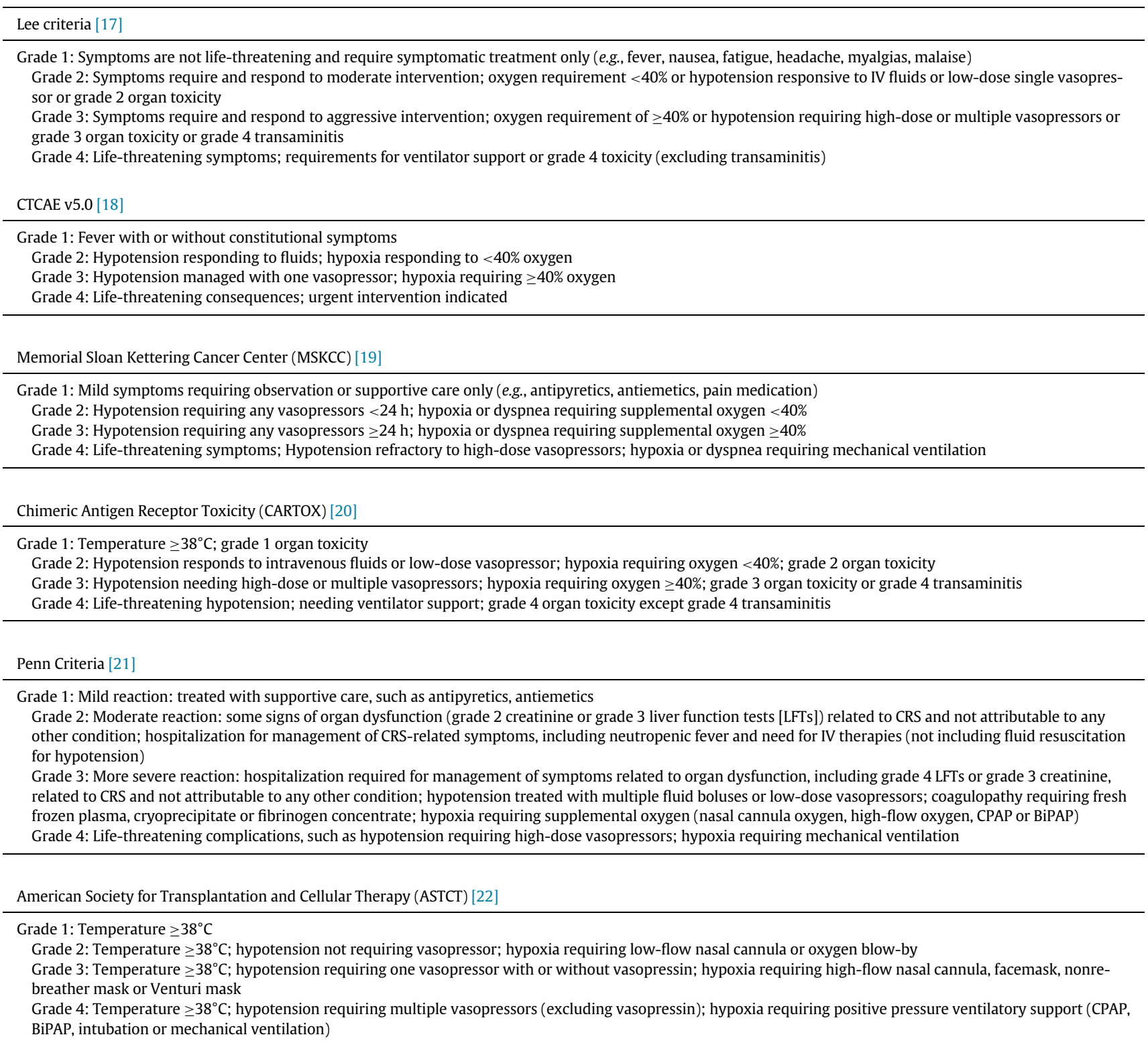

criteria (see Table 1). This is important, as the use of vasopressors or respiratory support is based on the clinical judgment of the physician, which may vary and thus lead to individual bias in CRS grading.

The presence of other organ function abnormalities is included in some, but not all, CRS grading systems. Other organ abnormalities could be reported either as separate AEs with no relationship to CRS or as preferred terms encompassing CRS. Therefore, it is important to clarify whether the definition of CRS should consider including these abnormalities to capture the full extent of CRS and minimize the risk of underdocumenting or underreporting. Additionally, if a therapeutic modality has the potential to cause clinically severe CRS that requires treatment with fluids, vasopressors, supplemental oxygen and anti-cytokine therapy, then initial low-grade events related to these manifestations should be assumed to be part of that spectrum and defined as CRS. Although there are a variety of published manuscripts, descriptions and adapted grading criteria and management strategies for CRS [22], it is noted that published definitions and grading criteria do not readily articulate the distinctions among CRS and other clinical entities that may have overlapping symptoms and temporality (e.g., IRR, macrophage activation syndrome [MAS]/HLH).

Given the current variations in defining and reporting CRS, the working group feels an urgent need to harmonize the grading, collecting and reporting of CRS. Below are the working group proposals.

\section{Alignment on Defining and Grading CRS}

The American Society for Transplantation and Cellular Therapy (ASTCT) defines CRS as "a supraphysiologic response following any immune therapy that results in the activation or engagement of endogenous or infused $\mathrm{T}$ cells and/or other immune effector cells. Signs and symptoms can be progressive, must include fever at the onset, and may include hypotension, capillary leak (hypoxia), and end organ dysfunction" [22]. ASTCT's definition for CRS represents an opportunity for alignment and prioritization of grading of clinically relevant events and can be inclusive of currently available and 
Table 2

Principle components for defining CRS.

$\begin{aligned} & \text { Principles } \\ & \text { Therapeutic modality }\end{aligned}$
$\begin{aligned} & \text { Considerations } \\ & \text { Therapeutic schedule } \\ & \text { therefore, the definition of CRS may evolve. } \\ & \text { The onset of CRS and severity can differ based on treatment administration (i.e., one-time infusion vs. multiple infusions). Kinetics of } \\ & \text { CRS may differ by both disease state and therapeutic platform (e.g., cellular products vs. T cell engagers). } \\ & \text { The timing of development of CRS depends on patient-, disease- and treatment-related factors. In the setting of CAR T cells, in vivo } \\ & \text { expansion of CAR T cells is associated with the onset and maximum severity of CRS. A reasonable temporal relationship to the } \\ & \text { therapy must be present. } \\ & \text { A suspected diagnosis of CRS should be made based on clinical signs and symptoms. Hallmarks of CRS are fever with or without } \\ & \text { hypotension and hypoxia; however, symptoms of CRS are not unique and overlap with other toxicities. Careful evaluation is } \\ & \text { required to ensure that the symptoms are associated with the cancer therapy, and other information such as blood cultures, fever } \\ & \text { workup, etc., should be collected to assist in the differential. } \\ & \text { Baseline assessment of inflammatory markers can assist in comparing with increased levels after treatment. Laboratory evaluation } \\ & \text { including C-reactive protein and ferritin are routinely available. Other cytokine level assessments (IL-6, IL-1, IL-8, TNF } \alpha \text { and IFN } \gamma \text { ), } \\ & \text { if available, can be helpful in further characterizing this syndrome retrospectively (unless available in real time). } \\ & \text { CRS implies the toxicity may be effectively treated with anti-IL- } 6 \text { therapy or other cytokine-directed therapies given in conjunction } \\ & \text { with corticosteroids, depending on the type of immunotherapy. }\end{aligned}$
$\begin{aligned} & \text { Laboratory evaluation } \\ & \text { Interventional care }\end{aligned}$

emerging immunotherapies, with some considerations as noted below.

Although each CRS grading scale in Table 1 has advantages and limitations, the working group supports the use of a harmonized definition and grading scale as well as collection of common data elements within and across development programs. An informal sponsor survey indicated that of eight sponsors, seven are using/ planning to use ASTCT criteria for new protocols (see Supplement for Survey Summary). Several sponsors indicated that some development programs have been underway before the release of the ASTCT 2019 grading criteria, and CTCAE and Lee Criteria 2014 were predominantly being used to grade CRS. This is likely driven by efforts to simplify the characterization and categorization of the severity of CRS in the ASTCT criteria. Some limitations exist, such as the overlapping nature of oxygen requirements between grade 1 and grade 2 hypoxia due to the reliance on the oxygen delivery method and exclusion of end organ toxicities that result from CRS (e.g., renal or hepatic injury). Furthermore, the use of proactive premedication (e.g., corticosteroids) may limit or minimize the cascade of signs and symptoms of CRS, such as fever, hypotension and hypoxia, which is used as a defining characteristic of CRS in the ASTCT 2019 definition.

Because these guidelines have primarily been developed based on the clinical experience with CAR T cell therapy, they may prove, with additional clinical experience, to be incomplete for all cancer immunotherapies and may need to be revised as new data become available from existing and novel therapies [22]. As such, it is important that data collection is aimed at more than meeting the requirements of any one grading system. Therefore, establishing core principles for defining CRS that consider the therapeutic modality, symptom manifestation, timing and response to intervention will be important to enable flexibility and maximize utility of a harmonized definition for CRS to adequately assess safety profiles of therapeutics being offered to patients (Table 2).

\section{Strategy for Assessing CRS over the Course of a Clinical Development Program}

The characterization of CRS for a given experimental therapeutic in the course of a clinical development program is crucial to ensure the correct diagnosis and management of toxicity to help maximize treatment benefit. During the development of protocols for safety data collection and monitoring strategies as they relate to CRS, consideration should be given to how toxicities will be identified and managed in routine clinical care. Recognizing the association between the immunotherapeutic agent and CRS will inform the framework on how best to collect these data.
The collection of a broad dataset for characterizing CRS is resource intensive for both sponsors and investigators; however, assessing the risk of an IRR or CRS during preclinical and early clinical development of a new therapy will help gauge the robustness of data collection required during development to characterize the potential risk of CRS (Figure 1). The robustness of data collection can be assessed using a decision tree approach, which includes (1) an initial assessment of the risk of IRR or CRS based on mechanistic models and preclinical assessment; (2) biomarker and clinical data collection; and (3) iterative review of aggregate data to make an informed decision regarding CRS designation.

If there is a low risk or no risk of IRR or CRS based on mechanistic models, known class effects and nonclinical data, "LOW/NO" guidelines would be followed (Figure 1). In this instance, standard AE reporting and no upfront cytokine or other biomarker data collection would be recommended initially. With ongoing frequent safety data review and consideration for inclusion of cytokine and biomarker data collection, the data collection plan should be adapted if the clinical data are suggestive of potential IRR or CRS toxicity.

For therapeutic classes that are known to be associated with CRS or at particularly high risk for inducing CRS based on mechanism of action or preclinical data, the implementation of a dedicated clinical and safety monitoring plan may be required from the onset. The potential risks of IRR and CRS should be defined in the Investigator Brochure and protocol for the first-in-human trial, with a dedicated case report form (CRF) for IRR and/or CRS that collects the associated signs and symptoms. In addition, special preparation may be warranted as part of the protocol such as specific site training on CRS and the requirement of certain clinical interventions (e.g., inpatient monitoring, intensive care unit [ICU] availability, and readily available tocilizumab). In most circumstances, it is recommended that physicians report either IRR or CRS as the Medical Dictionary for Regulatory Activities (MedDRA) preferred term until human data at the population level (e.g., aggregate data in the clinical trial) are available. If there is evidence at the population level of cytokine-driven clinical signs and symptoms, increase in CRS biomarkers such as IL-6 or responsiveness to tocilizumab or other cytokine-directed therapies, it would be concluded that CRS is an identified risk and can then be characterized accordingly and allow proper clinical management. Lack of such evidence (e.g., response to IL-6 directed agents) may suggest that the reaction is a manifestation of IRR or hypersensitivity but should not exclude the possibility of CRS based on further exploration and clinical assessments.

As more data are collected in a harmonized fashion, the field can better decide at which point and with which factors an event is determined to be a high-grade IRR versus a low-grade CRS. Understanding if there are implications on patient management will be important. 


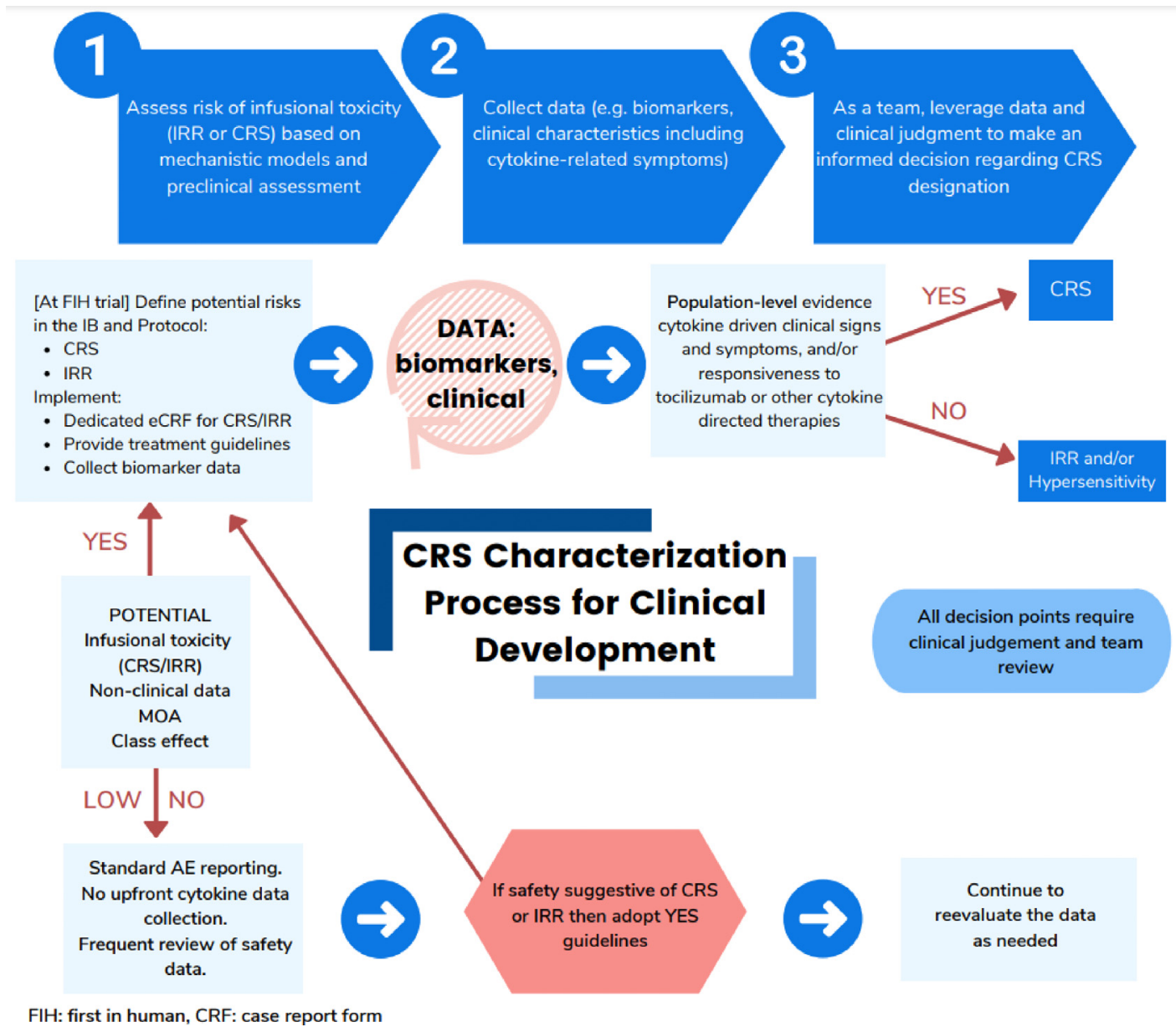

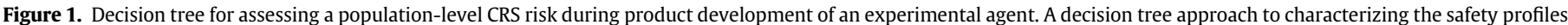

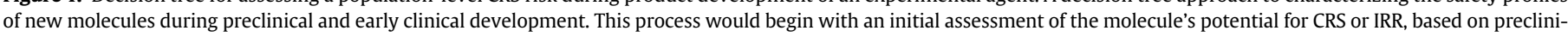
cal data, mechanism of action and experience with other agents in the same therapeutic class. (Color version of figure is available online.)

\section{Harmonized Data Elements for Characterizing CRS}

With the evolution of defining and grading CRS in the field, there is an opportunity for the medical community to ensure that the appropriate data elements are collected to allow derivation with different grading systems. Collection of common data variables using aligned protocols will be important to enable comparison with different therapies in the future. Early in the clinical development of a novel therapy, it is important to collect individual signs and symptoms associated with each case of CRS, since the definition of CRS has evolved and is likely to continue to evolve as more experience is gained with immunotherapies. A suspected diagnosis of CRS will most likely be based on clinical signs and symptoms, such as fever, hypotension and hypoxia [24]. However, the collection of all individual signs and symptoms consider to be associated with CRS as well as certain data variables, such as laboratory assessments, cytokine profiles and biomarkers, will be important for future retrospective analyses to assess the relationship of certain signs and symptoms with CRS, the severity of CRS, natural history of the event including response to therapy, or the identification of predictive biomarkers. CRS would generally be considered as an AE of special interest (AESI) if there are CRS reports in early clinical studies of the immunotherapy product or with products of the same class. A confirmatory diagnosis could be made at a later date and in the context of the evolution of clinical symptoms and cytokine data or response to cytokine-directed interventions (see section "Consistent Method for Recording and Reporting CRS Events").
Table 3 outlines key data elements driven largely in part by ASTCT 2019. Review of key data variables from published severity scales would inform the components of a dedicated CRF for CRS. These represent minimum data collection elements, and sponsors may capture additional variables. Comprehensive data capture will be critical to facilitate new iterations of grading criteria and past criteria to ensure the safe monitoring and administration of T cell-engaging immunotherapies.

Vital sign assessment should include body temperature, pulse (heart rate), blood pressure and oxygen saturation. It is important to note that ASTCT grading depends on the use of supplemental oxygen or positive pressure ventilation and the use of vasopressors. Because the criteria to use these interventions are not standardized, some bias could be introduced into the grading of CRS. Once CRS is further characterized, biomarker testing can be reduced to key time points and biomarkers. Capturing these core data elements may be important for drug label descriptions and management guideline development.

Additional laboratory tests to consider among patients who experience a more severe manifestation of CRS without initial response to interventional therapy can include fibrinogen and complete blood counts (if not already included in the routine hematologic laboratory assessments), triglycerides, and a bone marrow biopsy. The latter would be necessary to confirm the diagnosis of MAS/HLH, which likely has a worse prognosis and may warrant additional therapies.

In the setting of CAR T cell therapy, one important determinant associated with CRS and its severity is the in vivo expansion of these cells after infusion. While to-date treatment guidelines are based on 
Table 3

Harmonized collection of discrete data elements.

\begin{tabular}{|c|c|}
\hline Parameter & Data collection \\
\hline Signs and symptoms & $\begin{array}{l}\text { Minimum signs and symptoms to collect } \\
\text { include fever, nausea, chills, vomiting, } \\
\text { diarrhea, confusion, dizziness, dyspnea, } \\
\text { tachycardia, headache, hypotension, hyp- } \\
\text { oxia, lymphadenopathy, hepatosplenome- } \\
\text { galy; but the eCRF should allow an } \\
\text { investigator to enter any symptom thought } \\
\text { to be a CRS symptom } \\
\text { Date/time onset (e.g., x hour[s] after infu- } \\
\text { sion of dose); initial grade; maximum } \\
\text { grade; date/time resolution; intervention; } \\
\text { outcome }\end{array}$ \\
\hline Hypotension management & $\begin{array}{l}\text { No intervention required, blood pressure val- } \\
\text { ues, intravenous fluids, use of vasopressors } \\
\text { and dose, start/stop date of treatment, } \\
\text { duration of treatment }\end{array}$ \\
\hline Hypoxia management & $\begin{array}{l}\text { No oxygen supplementation required, regu- } \\
\text { lar flow nasal cannula, high-flow nasal can- } \\
\text { nula, facemask, nonrebreather mask, or } \\
\text { Venturi mask; positive pressure ventilatory } \\
\text { support (CPAP, BiPAP, intubation, mechan- } \\
\text { ical ventilation) }\end{array}$ \\
\hline Organ toxicity & $\begin{array}{l}\text { Liver function tests, creatinine, amylase, } \\
\text { lipase, rash, neurotoxicity, cardiac, pulmo- } \\
\text { nary, renal, hepatic toxicities }\end{array}$ \\
\hline Cytokines & $\begin{array}{l}\text { IL-6, IL-1, IL-8, TNF } \alpha \text { and IFN } \gamma \text { are recom- } \\
\text { mended as a core cytokine panel, if avail- } \\
\text { able and considered in a research setting }\end{array}$ \\
\hline Other laboratory assessments & $\begin{array}{l}\text { Routine hematology analysis, including com- } \\
\text { plete blood count and differential, serum } \\
\text { chemistries, coagulation factors, ferritin, C- } \\
\text { reactive protein }\end{array}$ \\
\hline Care setting & $\begin{array}{l}\text { Admitted to hospital or ICU; duration, includ- } \\
\text { ing distinction between ICU and non-ICU } \\
\text { duration }\end{array}$ \\
\hline Intervention for management & $\begin{array}{l}\text { Tocilizumab or other cytokine-directed ther- } \\
\text { apy administered for management, as well } \\
\text { as corticosteroids or other supportive care, } \\
\text { such as antipyretics, and type of prophy- } \\
\text { laxis, if any; if applicable, permanent dis- } \\
\text { continuation of immunotherapy or ability } \\
\text { to rechallenge and administer therapy }\end{array}$ \\
\hline
\end{tabular}

symptoms, it is important to capture the laboratory value information, including cytokine biomarkers, as laboratory values and cytokines help improve our understanding of the pathophysiology and may inform future development of management guidelines. Although there are currently no commercially available assays to determine expansion and persistence of CAR T cells, and real-time cytokine analysis is also not typically available, correlative analyses in the context of clinical trials may allow retrospective analyses to interrogate CRS cases and direct future guidelines for toxicity management.

With CAR T cell therapy, routine CRS assessment may range from daily CRS assessments immediately after infusion, to two to three times a week for the first 30 days after infusion, to help characterize the evolution of symptoms, development of additional toxicities, treatment and response to treatment. The timing and frequency of CRS assessments for T cell engagers may vary and depend on the pharmacokinetics and pharmacodynamics of the particular molecule and dosing schedule. Timing of sampling should be adapted to accommodate treatment cycles and protocol-defined scheduled visits.

\section{Consistent Method for Recording and Reporting CRS Events}

As described in Figure 1, identification and characterization of CRS early in a new experimental therapeutic's clinical development can be challenging owing to the heterogeneity in signs and symptoms and similarity of CRS signs and symptoms to those of other AEs, such as IRR or infection, as noted earlier. A hypothetical case is shown in Figure 2. A patient treated with a $T$ cell engager experiences several AEs. Initially, the patient presents with a fever of $40.1^{\circ} \mathrm{C}$ lasting $6 \mathrm{~h}$ that is accompanied by hypotension responsive to a 1-liter fluid bolus. The fever and hypotension are CTCAE grade 3 . The next morning, liver function test (LFT) increases are noted (grade 4), and later that day, the patient has a brief generalized seizure that is self-limited, lasting less than a minute (grade 2). Grade 2 CRS is diagnosed [22]. It is important for all these AEs to be captured into the CRF and independently reported for characterization of the range and severity of signs and symptoms constituting the grade 2 CRS. Although all of these may precede the investigator diagnosing CRS, all AEs should be captured into the $\mathrm{CRF}$ and independently reported. Independent $\mathrm{AE}$ reporting is critical, and clinical investigators should carefully describe all events that are suggestive of potential CRS. This will enable pharmacovigilance experts to evaluate relevant, linked events and code them as CRS, as appropriate.

In the example case, the event of fever precedes the diagnosis of CRS and would be captured into the AE database independently and graded independently, as the differential diagnosis for the fever could include not only CRS, but other potential etiologies such as IRR and infection. Additional events such as the increase in LFTs and seizure are attributable to the CRS, but could also be recorded as independent AEs into the database. Once CRS is diagnosed and recorded as an AE in the trial database, the signs and symptoms indicative of CRS ideally would be linked to the CRS event.

We propose a comprehensive method to capture all the events and link those AEs that are signs and symptoms of CRS to the CRS event, such that CRS is the AE, but the symptoms (fever, LFT increase, seizure) that are AEs in themselves are attributable to CRS and are linked to the CRS event (Figure 2, "Link Events to CRS"). For instance, one way is to flag each AE that is related to CRS and link it to the specific CRS event. This will allow a more qualitative analysis of CRS, as CRS can manifest in a variety of organs including hepatic, renal and neural system. This method would also allow the optionality of reporting all AEs, CRS and the specific organ toxicity separately or allow collapsing of the CRS-related events to a single AE. Given the importance of central nervous system (CNS)-related toxicity with $\mathrm{T}$ cell therapies, it is recommended that ICANS (immune effector cellassociated neurotoxicity syndrome) events be captured and scored separately. In the case described, any seizure would be captured as a grade $\geq 3$ ICANS.

Without data collection standards, several outcomes in terms of data capture may arise. For instance, one possible method is that all the signs and symptoms that are attributable to CRS could be collapsed into the AE preferred term of CRS. Once the investigator identifies CRS, as part of data cleaning, the fever, LFT increase and even seizure events could be accounted for by CRS, and only the CRS event is reported (Figure 2, "Collapse Events to CRS"). However, this method would lead to the loss of actionable information that may be useful for retrospective application of future CRS diagnostic or grading criteria and for physicians and patients.

As described in Table 3, additional information would be captured including use of concomitant medications (e.g., tocilizumab or other cytokine-directed therapy, oxygen, vasopressors, corticosteroids) and specific interventions (e.g., method of oxygen delivery, mechanical intervention, intravenous fluids). In our example case, the use of intravenous fluids and not vasopressors define a grade 2 CRS event. Although these items may be collected in other parts of the electronic data capture record, it is important that they are easily linked to a specific CRS event, as CRS grading is dependent on these interventions in most classification systems. In addition, some grading systems can lead to downgrading of events. As an example, liver function laboratory values may increase 


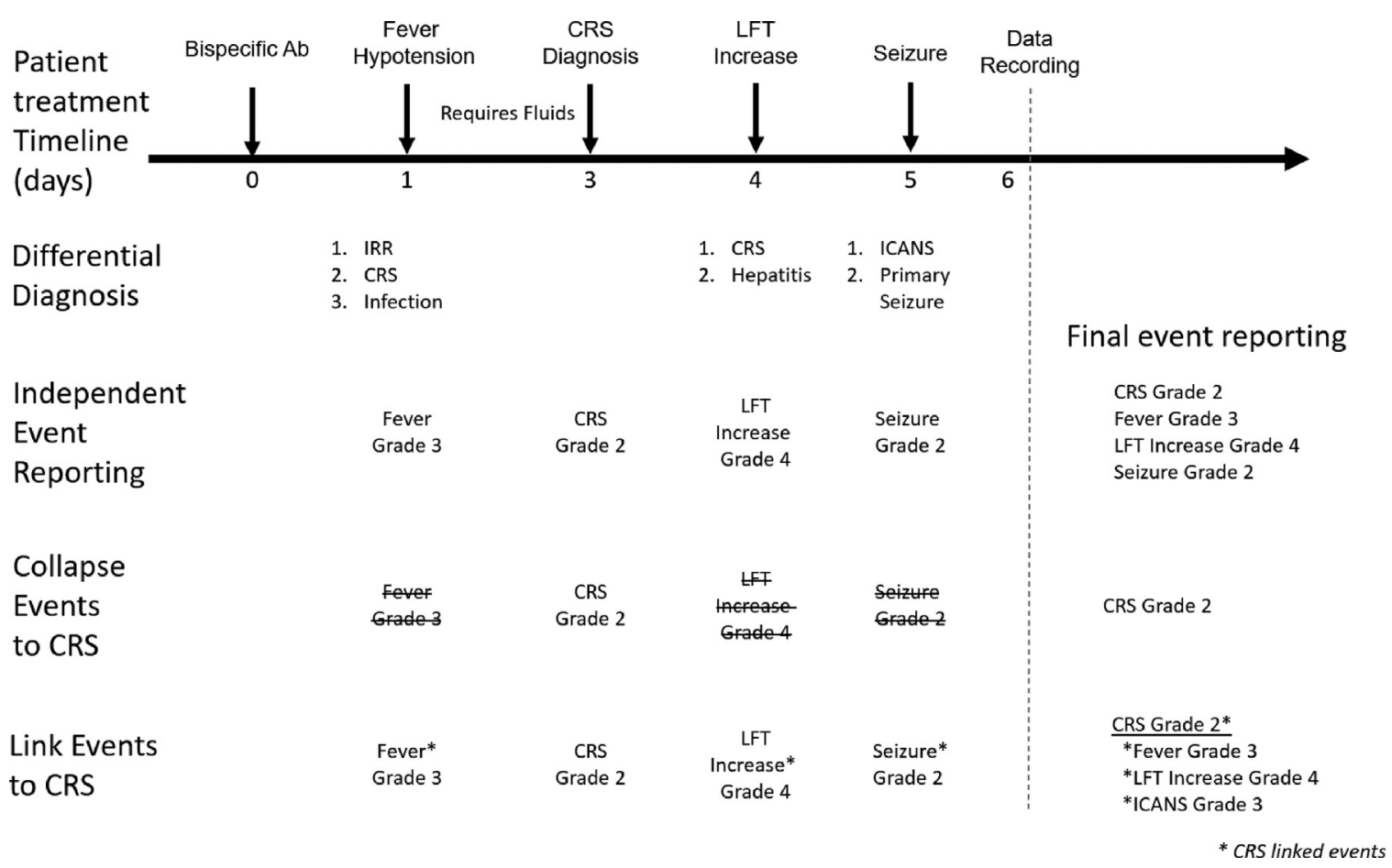

Figure 2. Patient treatment timeline and event reporting after immunotherapy administration.

transiently and meet the criteria for CTCAE grade 4 CRS based on these laboratory changes; however, this increase will only meet the definition of a grade 2 CRS by ASTCT criteria if it is not accompanied by clinically significant changes in blood pressure or oxygen requirement.

\section{Conclusions}

Cytokine release syndrome is commonly seen with newer immunotherapies, such as T cell engagers and CAR T cells, and presents as a range of signs and symptoms, most commonly fever, hypotension and hypoxia. There are currently several different scales used to grade the severity of CRS, and therefore, it is important to standardize the grading to ensure consistency in how data are collected and presented and to better distinguish CRS from other clinical entities with overlapping symptoms. Ideally, all investigators would commit to a harmonized data collection approach using a dedicated CRS eCRF with data elements identified in Table 3 as a guide. Moreover, this working group outlined several actionable proposals for deployment in early clinical development programs of emerging immunotherapies. To improve alignment on defining CRS, clinical programs could establish core principles that consider the therapeutic modality, symptom manifestation, timing, and response to intervention as part of a harmonized definition of CRS. A strategy for assessing CRS over the course of a clinical development program that takes into account an initial assessment of the risk of IRR or CRS, biomarker and clinical data collection, and an ongoing review of data is needed to make an informed decision of CRS designation. In regard to data, harmonized data elements for characterizing CRS need to be determined, as comprehensive data capture will allow for easier adaptation of one grading scale to another. Lastly, a consistent method for recording and reporting CRS events is necessary to simultaneously delineate the individual signs and symptoms of CRS as well as to characterize the CRS event as its own entity. In particular, the previous experience with the study of immune-related AEs due to checkpoint inhibitors emphasized that, when evaluating CRS events, alignment of important data elements and a more comprehensive understanding of AEs early in clinical development can support ongoing pharmacovigilance and real-world data collection to enable further characterization of these events in the postmarketing setting.

As our clinical understanding of CRS and other clinical entities associated with these types of therapies evolves, a harmonized approach for defining, characterizing and reporting CRS in patients receiving immunotherapies is necessary to support evidence-based monitoring and management of novel toxicities; facilitate and harmonize the assessment and communication of risk-benefit profiles with regulatory agencies, the clinical community, and the public; and improve patient care and outcomes. Furthermore, such an approach can also support retrospective analyses to compose new iterations of grading criteria and clinical guidelines, to ensure the safe monitoring and administration of $\mathrm{T}$ cell engaging immunotherapies.

\section{Disclaimer}

This article reflects the views of the authors and should not be construed to represent FDA's views or policies.

\section{Conflicts of Interest}

B.M. is an employee of Genentech and owner of Roche stock. A.S.Y. is an employee of Xencor and owner of Xencor stock. C.D.B. is an employee of Amgen and owner of Amgen stock. M.M.H. has received grant funding for research from Allovir, Amgen, Astellas, Gamida Cell Ltd., Genentech Inc., Magenta Therapeutics, Medac GmbH, OncImmune, and Vor Biopharma. C.M. is an employee of BMS and owner of BMS and Amgen stocks. P.K.M. is an employee of Amgen and owner of Amgen stock. E.O. is an employee of Regeneron. R.R. is an employee of Bristol Myers Squibb. F.V. is an employee of Bristol Myers Squibb. T. Y. is an employee of Amgen and owner of Amgen stock. All other authors have no disclosures to report. 


\section{Acknowledgment}

The authors thank Yifang Eva Pan for writing support and editorial assistance and Joe Patterson (Friends of Cancer Research) for graphical assistance.

\section{Supplementary materials}

Supplementary material associated with this article can be found in the online version at doi:10.1016/j.jcyt.2022.01.004.

\section{References}

[1] Xin Yu J, Hubbard-Lucey VM, Tang J. Immuno-oncology drug development goes global. Nature reviews. Drug discovery 2019;18:899-900.

[2] Khadka RH, Sakemura R, Kenderian SS, Johnson AJ. Management of cytokine release syndrome: an update on emerging antigen-specific $\mathrm{T}$ cell engaging immunotherapies. Immunotherapy 2019;11:851-7.

[3] Murthy H, Iqbal M, Chavez JC, Kharfan-Dabaja MA. Cytokine Release Syndrome: Current Perspectives. ImmunoTargets Ther 2019;8:43-52.

[4] Maus MV, et al. Society for Immunotherapy of Cancer (SITC) clinical practice guideline on immune effector cell-related adverse events. J. Immunother. cancer 2020;8.

[5] Santomasso BD, et al. Management of Immune-Related Adverse Events in Patients Treated With Chimeric Antigen Receptor T-Cell Therapy: ASCO Guideline. J. Clin Oncol. Off. J. Am. Soc. Clin. Oncol. 2021;39:3978-92.

[6] Doessegger L, Banholzer ML. Clinical development methodology for infusionrelated reactions with monoclonal antibodies. Clin. Transl. Immunol. 2015;4:e39.

[7] Roselló S, et al. Management of infusion reactions to systemic anticancer therapy: ESMO Clinical Practice Guidelines. Ann. Oncol. 2017;28:iv100-18.

[8] Bupathi M, et al. Incidence of infusion reactions to anti-neoplastic agents in early phase clinical trials: The MD Anderson Cancer Center experience. Invest. New Drugs 2017;35:59-67.
[9] Bernardez, B. et al. Real world of infusion related reactions in an oncology outpatient clinic. (2018).

[10] Chung $\mathrm{CH}$, et al. Cetuximab-induced anaphylaxis and IgE specific for galactose$\alpha$-1, 3-galactose. N. Engl. J. Med. 2008;358:1109-17.

[11] Farshi, E. Cytokine Storm Response to COVID-19 Vaccinations. (2021).

[12] Giavridis T, et al. CAR T cell-induced cytokine release syndrome is mediated by macrophages and abated by IL-1 blockade. Nat. Med. 2018;24:731-8.

[13] Shimabukuro-Vornhagen A, et al. Cytokine release syndrome. J. Immunother. cancer 2018;6:56.

[14] Sampson HA, et al. Second symposium on the definition and management of anaphylaxis: summary report-Second National Institute of Allergy and Infectious Disease/Food Allergy and Anaphylaxis Network symposium. The Journal of allergy and clinical immunology 2006;117:391-7.

[15] Maude SL, Barrett D, Teachey DT, Grupp SA. Managing cytokine release syndrome associated with novel T cell-engaging therapies. Cancer J 2014;20:119.

[16] Le RQ, et al. FDA Approval Summary: Tocilizumab for Treatment of Chimeric Antigen Receptor T Cell-Induced Severe or Life-Threatening Cytokine Release Syndrome. Oncologist 2018;23:943-7.

[17] Lee DW, et al. Current concepts in the diagnosis and management of cytokine release syndrome. Blood 2014:124:188-95.

[18] National Cancer Institute. Common Terminology Criteria for Adverse Events (CTCAE) Version 5.0. https://ctep.cancer.gov/protocoldevelopment/electronic_applications/docs/CTCAE_v5_Quick_Reference_8.5x11.pdf (2017).

[19] Park JH, et al. Long-Term Follow-up of CD19 CAR Therapy in Acute Lymphoblastic Leukemia. N. Engl. J. Med. 2018;378:449-59.

[20] Neelapu SS, et al. Chimeric antigen receptor T-cell therapy - assessment and management of toxicities. Nat. Rev. Clin. Oncol. 2018;15:47-62.

[21] Porter D, Frey N, Wood PA, Weng Y, Grupp SA. Grading of cytokine release syndrome associated with the CAR T cell therapy tisagenlecleucel. J. Hematol. Oncol. 2018;11:35.

[22] Lee DW, et al. ASTCT Consensus Grading for Cytokine Release Syndrome and Neurologic Toxicity Associated with Immune Effector Cells. Biol. blood marrow Transplant. J. Am. Soc. Blood Marrow Transplant. 2019;25:625-38.

[23] Davila ML, et al. Efficacy and toxicity management of 19-28z CAR T cell therapy in B cell acute lymphoblastic leukemia. Sci. Transl. Med. 2014;6. 224 ra25.

[24] Wei J, et al. The model of cytokine release syndrome in CAR T-cell treatment for B-cell non-Hodgkin lymphoma. Signal Transduct. Target. Ther. 2020;5:134. 